\title{
Uso de situaciones problema para desarrollar el pensamiento matemático
}

Juan Arturo Jiménez Parra*
Fredy Jesid Herrera Reyes
Publio Suárez Sotomonte

Artículo de Investigación

Fecha de Recepción: 10 noviembre 2017.

Fecha de Aprobación: 15 abril 2018.

\section{Resumen}

En el diagnóstico de la investigación, se identificó que la mayoría de estudiantes de los grados tercero y quinto de la I.E. Antonio Ricaurte de Santana - (Boyacá), presentaron debilidades en las diferentes competencias y componentes que evalúa el ICFES. Se reconoció, que los docentes no orientan algunos contenidos y conceptos necesarios para que los estudiantes desarrollen competencias y aprendizajes, que evalúan las Pruebas Saber. Teniendo en cuenta esta situación, se centralizó la investigación en la reflexión docente para (re)significar sus prácticas de aula. También se estableció como situación de aprendizaje clave para mejorar en los estudiantes el desarrollo de su pensamiento matemático, la resolución de situaciones problema. El método utilizado fue la investigación acción, adoptando el modelo de Kemmis, y el taller investigativo como técnica, con la participación de cuatro docentes de primaria, quienes orientan el área de matemáticas. Con las reflexiones realizadas, se elaboraron planes de aula/ clase, se implementaron secuencias didácticas y centros de aprendizaje, lo que permitió integrar los contenidos de los pensamientos que poco se orientaban y trabajar de manera transversal diferentes pensamientos, fortaleciendo en los estudiantes estrategias de comprensión, desarrollo de competencias matemáticas y construcción de conceptos.

Palabras clave: reflexión docente, situaciones problema, pensamiento matemático, (re)significación, prácticas de aula.
* Institución Educativa Técnica Antonio Ricaurte, -Boyacá- (Colombia). juanjimeneztutorpta2.0@ gmail.com

** Institución Educativa Técnica La Laja, -Boyacá(Colombia).

fredyherrera987@gmail.com *** Universidad Pedagógica y Tecnológica de Colombia, -Boyacá- (Colombia). publio.suarez@uptc.edu.co

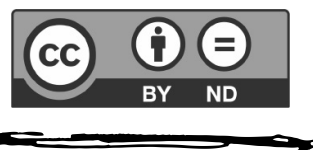




\section{Introducción}

En el diagnóstico de la investigación, se identificó con el análisis de los resultados en el área de matemáticas de las pruebas Saber 2014, 2015 y 2016 (ICFES), de las pruebas Egma y Diagnósticas 2015 (PTA 2.0 - MEN), y las pruebas Supérate 2015 y 2016 (MEN); que la mayoría de estudiantes de los grados tercero y quinto de la Institución Educativa Técnica Antonio Ricaurte (IEAR), presentaron debilidades en las diferentes competencias (comunicación, razonamiento, resolución de problemas) y componentes (numérico - variacional, geométrico - métrico, aleatorio), que evalúa el ICFES. Además, se reconoció, a través de la observación en el aula y la revisión de los planes de área, que los docentes no orientaban en su totalidad algunos contenidos y conceptos necesarios para que los estudiantes desarrollen competencias y aprendizajes, que sí se están evaluando en las pruebas Saber, como son los relacionados con los pensamientos: variacional, espacial y aleatorio, que se referencian en los informes por colegio 2015 y 2016 del “Siempre día E” (MEN 2015a, 2016a).

Frente a esta situación, con la construcción del estado del arte de la investigación, se reconocieron algunos aspectos didácticos de los procesos de enseñanza y aprendizaje de las matemáticas relevantes para el aprendizaje de los estudiantes, por lo cual se determinó que la investigación se debería llevar a cabo en la didáctica de la matemática tipo $C$, centrada en el docente. Según D’Amore, Fandiño, Marazzani y Sbaragli (2007), para algunos investigadores el docente "...es considerado mucho más importante en el proceso de enseñanza-aprendizaje de la matemática, dado que el estudiante no accede normalmente al saber, sí que lo hace a través de la mediación del docente que reconstruye una matemática de aprender para él” (p. 25).

También, se identificó que una de las estrategias para fortalecer el desarrollo de las competencias matemáticas en los diferentes tipos de pensamiento matemático, es el uso de las situaciones problema, la cual difiere de los conceptos de ejercicio y problema; se trata de una "...situación de aprendizaje concebida de manera tal que los estudiantes no pueden resolver la cuestión por simple repetición o aplicación de conocimientos o competencias adquiridas sino que se necesita la formulación de nuevas hipótesis." (D'Amore, 2006, p. 183).

Con el fin de reconocer la validez que dan los docentes a las anteriores afirmaciones y mejorar los aprendizajes de los estudiantes, surgió la pregunta de investigación: ¿Qué aspectos relevantes se reconocen con las reflexiones de los docentes en la aplicación de situaciones problema para desarrollar el pensamiento matemático en sus estudiantes?, para responder a este interrogante se planteó como objetivo general caracterizar los aspectos más relevantes en la reflexión que hacen los docentes sobre sus prácticas de aula al implementar situaciones problema para desarrollar el pensamiento matemático en los estudiantes de tercero y quinto de primaria de la sede urbana. 
La investigación se desarrolló en la IEAR, ubicada en el municipio de Santana, que pertenece a la provincia de Ricaurte Bajo, en el departamento de Boyacá; tiene una población de 5500 habitantes, la mayoría en el área rural, dedicados a la producción y comercialización de panela. El equipo de docentes acompañados se caracteriza por tener un alto sentido de pertenencia con el municipio y la I.E., se cuenta con un alto porcentaje de profesores oriundos de este pueblo, quienes demuestran interés y compromiso por mejorar los procesos de enseñanza y de aprendizaje. La población estudiantil se caracteriza principalmente por ser emocionales y participativos, una parte proviene de familias con escaso nivel académico y disfuncionales, presentando agresividad entre pares, desacato de normas, e irresponsabilidad académica (IEAR, 2017).

Como punto de partida de la metodología, se estableció la investigación con docentes para reflexionar sobre la acción en sus prácticas de aula, siguiendo las afirmaciones de Perrenoud (2007), "reflexionar sobre la acción [...] es tomar la propia acción como objeto de reflexión” (p. 29), y (re)significar de esta manera su quehacer pedagógico en torno a los procesos de enseñanza y aprendizaje de las matemáticas.

El método que se siguió con la intención de transformar la práctica educativa en un proceso crítico de intervención y reflexión, fue la investigación acción. Se adoptó el modelo de Kemmis (1988), integrado por cuatro fases interrelacionadas: planificación, acción, observación y reflexión (Murillo, 2011). En este proceso investigativo, se dio gran importancia a la reflexión docente (Jiménez, 2005). Siendo esta la base para caracterizar y estructurar los aspectos más relevantes sobre sus prácticas de aula; como técnica prioritaria de recolección de información, análisis y planeación, se usó el taller investigativo, en el cual participaron tres docentes de grado tercero y una docente de grado quinto, quienes orientan el área de matemáticas en la sede primaria urbana de la I.E.

Este taller se desarrolló teniendo en cuenta las fases propuestas por Quintana (2006), “(1) encuadre, (2) diagnóstico, (3) identificación, valoración y formulación de las líneas de acción, (4) estructuración y concertación del plan de trabajo" (p. 72). También se tuvo en cuenta, en la fase de descripción del problema, la revisión documental de los libros institucionales, como el Proyecto Educativo Institucional y los Planes de Área, y la observación en el aula sobre las prácticas docentes.

Con las reflexiones realizadas en torno al uso de las situaciones problema, se actualizó el plan de área de matemáticas integrando los contenidos de los pensamientos que poco se trabajaban; se construyeron planes de aula/clase basados en situaciones problema, lo que permitió trabajar con los estudiantes, de manera transversal, diferentes pensamientos; se realizaron centros de aprendizaje vinculados a las situaciones, fortaleciendo en los estudiantes
En este proceso investigativo, se dio gran importancia a la reflexión docente (Jiménez, 2005). Siendo esta

la base para caracterizar y estructurar los aspectos más relevantes sobre sus prácticas de aula 
el desarrollo de competencias matemáticas y la construcción de conceptos. Las docentes dieron gran importancia al uso de estrategias de comprensión y el desarrollo de las actividades en secuencia didáctica para la resolución de situaciones problema. Los estudiantes estuvieron motivados en las actividades e interesados en dar respuestas a las situaciones problemáticas. También, se resalta la importancia de contextualizar las situaciones para que los estudiantes le den sentido y significado a su aprendizaje, y se concluye que un proceso crítico y reflexivo es uno de los caminos para intentar la transformación educativa.

\section{Referentes teóricos}

Para la investigación, los elementos teóricos se agruparon en cinco grandes campos: (1) sobre la reflexión docente, (2) didáctica de la matemática, (3) situaciones problema, (4) resolución de problemas, y (5) pensamiento matemático. En cada uno se sintetiza los aspectos que se tuvieron como base en la investigación, y los conceptos que guían el proceso.

\section{Sobre la reflexión docente}

La transformación educativa hoy día exige de investigaciones que salgan de lo común, donde se investigue con los docentes y no sobre ellos, para, como dice Cajiao (citado por Jiménez, 2005), "[...] estimular la reflexión sobre la práctica pedagógica y producir nuevos saberes sobre esa práctica [...]" (p. 12). Siguiendo esta idea, se estableció la investigación con docentes para reflexionar sobre aspectos relevantes en sus prácticas de aula cotidianas; a través de reuniones en comunidades de aprendizaje, donde se expresaron libremente las ideas y posiciones frente a los procesos de enseñanza y aprendizaje que llevaron a cabo.

En la investigación se resaltó la importancia del docente como profesional, para que, con autonomía y responsabilidad, se dirigieran a una verdadera práctica reflexiva sobre la acción; según Perrenoud (2007), la capacidad de reflexionar en la acción y sobre la acción '[...] está en el interior del desarrollo permanente, según la propia experiencia, las competencias y los conocimientos profesionales de cada uno" (p. 12).

\section{Acerca de la didáctica de la matemática}

La didáctica de la matemática, considerada como sinónimo de educación matemática por franceses y españoles, y como una subárea de la educación matemática por los anglosajones; tiene investigadores reconocidos como D'Amore (2007, 2006, 1997), de quien se retomaron varios elementos teóricos que se exponen a continuación, para contextualizar la investigación en los aspectos didácticos, epistemológicos y metodológicos.

Esta investigación se enfocó en una investigación de la didáctica de la matemática tipo $\mathrm{C}$, que se centra en el docente, quien es visto por los investigadores, como un ser esencial en el proceso de enseñanza y aprendizaje, 
el cual modela el saber académico para llevarlo a sus estudiantes, de manera que, de su asertividad en la transposición didáctica depende, en parte, la calidad del aprendizaje.

Dentro de los obstáculos para el aprendizaje, es claro que la actuación docente radica en los de naturaleza didáctica, por lo cual, la transposición didáctica es un elemento clave en el análisis de las reflexiones de los docentes.

La transposición didáctica consistiría entonces, desde el punto de vista del maestro, en el construir sus propias clases recabando de la fuente de los saberes, tomando en cuenta las orientaciones proporcionadas por las instrucciones y los programas (saber por enseñar), para adaptarlos a su propia clase: nivel de los estudiantes, objetivos perseguidos. La transposición didáctica consiste en el extraer un elemento de saber de su contexto (universitario, social,...) para (re) contextualizarlo en el contexto siempre singular, siempre único, de su propia clase (D'Amore, 2006, p. 181).

\section{Relevancia de las situaciones problema}

Como eje de análisis de la transposición didáctica, se tiene el uso de situaciones problema para el desarrollo del pensamiento matemático, definida como: "[...] situación de aprendizaje concebida de manera tal que los estudiantes no pueden resolver la cuestión por simple repetición o aplicación de conocimientos o competencias adquiridas sino que se necesita la formulación de nuevas hipótesis” (D’Amore, 2006, p. 183).

[...] una situación problemática y no un problema: el estudiante se halla frente a un problema al interior de una actividad más vasta, por lo que el resolutor domina el proyecto o, por lo menos, lo conoce y lo comparte con sus compañeros y con el maestro. Se trata por tanto de un verdadero y propio obstáculo para la prosecución de una actividad que en cambio se quiere proseguir y por lo tanto la motivación debe ser fuerte para que el estudiante tenga la necesidad y el deseo de recurrir a la creatividad, haciendo hipótesis, inventando soluciones ( $\mathrm{D}$ ' Amore, 2006, p. 184).

Según Borasi (citado en D’Amore, 2006), la situación problemática es "el contexto en el que tiene sentido el problema puesto" (p. 187). Situación problemática es el sistema de las competencias reales en las que puede imaginarse lo descrito por un texto y por su significado (semántica), al interior de las experiencias de cada sujeto (el sistema es específico para la situación dada).

\section{¿Por qué la Resolución de Problemas?}

Se sitúa la situación problema con su relación directa con la resolución de problemas, como estrategia potencial para el aprendizaje y el desarrollo del pensamiento matemático. Varios autores destacan la relevancia de la resolución de problemas en el
La transposición didáctica consistiría entonces, desde el punto de vista del maestro, en el construir sus propias clases recabando de la fuente de los saberes, tomando en cuenta las orientaciones proporcionadas por las instrucciones $\mathrm{y}$ los programas (saber por enseñar), para adaptarlos a su propia clase: nivel de los estudiantes, objetivos perseguidos. 
aprendizaje de las matemáticas, como Pólya (citado en D’Amore, 2006), "La resolución de problemas ha sido la espina dorsal de la enseñanza matemática desde la época del papiro de Rhind". Krygowska (citada en D'Amore, 2006) afirma que: "La resolución de problemas es la forma más eficaz no sólo del desarrollo de la actividad matemática de los estudiantes, sino también del aprendizaje de los conocimientos, de las habilidades, de los métodos y de las aplicaciones matemáticas"; y Duncker (citado en D’Amore, 1997) expone que: "un problema surge cuando un ser viviente tiene una meta pero no sabe alcanzarla" (p. 190).

\section{Desarrollodel pensamien- to matemático}

En los lineamientos curriculares del Ministerio de Educación Nacional (MEN, 1998) respecto a la formación matemática básica, el énfasis está en potenciar el pensamiento matemático mediante la apropiación de contenidos que tienen que ver con ciertos sistemas matemáticos. Tales contenidos se constituyen en herramientas para desarrollar, entre otros, el pensamiento numérico, el espacial, el métrico, el aleatorio y el variacional que, por supuesto, incluye al funcional (p. 16).

[...] provocar procesos de investigación que subyacen al razonamiento matemático; nos estamos refiriendo precisamente a los procesos del pensamiento matemático: la manipulación (exploración de ejemplos, casos particulares); la formulación de conjeturas (núcleo del razonamiento matemático, proponer sistemáticamente afirmaciones que parecen ser razonables, someterlas a prueba y estructurar argumentos sobre su validez); la generalización (descubrir una ley y reflexionar sistemáticamente sobre ella); la argumentación (explicar el porqué, estructurar argumentos para sustentar generalización, someter a prueba, explorar nuevos caminos) (MEN, 1998, p. 53).

Para el desarrollo del pensamiento matemático, es clave el estudio y análisis de situaciones problemáticas suficientemente complejas y atractivas, en las que los estudiantes mismos inventen, formulen y resuelvan problemas matemáticos. Para el MEN (2005):

La formulación, el tratamiento y la resolución de los problemas suscitados por una situación problema permiten desarrollar una actitud mental perseverante e inquisitiva, desplegar una serie de estrategias para resolverlos, encontrar resultados, verificar e interpretar lo razonable de ellos, modificar condiciones y originar otros problemas. Es importante abordar problemas abiertos donde sea posible encontrar múltiples soluciones o tal vez ninguna. También es muy productivo experimentar con problemas a los cuales les sobre o les falte información, o con enunciados narrativos o incompletos, para los que los estudiantes mismos tengan que formular las preguntas (p. 52).

\section{Metodología}

La investigación se cimentó en un enfoque de corte cualitativo, bajo el 
paradigma crítico social, que asume el conocimiento como una creación compartida a partir de la interacción entre el investigador y el investigado, en la cual, la subjetividad y la intersubjetividad se conciben como los medios e instrumentos para conocer las realidades humanas (Sandoval, 1996).

Dentro del enfoque cualitativo, en el paradigma crítico social, se encuentra el método que se lleva a cabo: la investigación acción, que en palabras de Kemmis (citado por Murillo, 2011) es:

[...] Una forma de indagación autorreflexiva realizado por quienes participan (profesorado, alumnado, o dirección por ejemplo) en las situaciones sociales (incluyendo las educativas) para mejorar la racionalidad y la justicia de: a) sus propias prácticas sociales o educativas; b) su comprensión sobre las mismos; y c) las situaciones e instituciones en que estas prácticas se realizan (aulas o escuelas, por ejemplo) (p. 4).

El modelo de Kemmis (citado por Murillo, 2011) se adoptó para llevar el proceso de investigación-acción:

El proceso lo organiza sobre dos ejes: uno estratégico, constituido por la acción y la reflexión; y otro organizativo, constituido por la planificación y la observación. Ambas dimensiones están en continua interacción, de manera que se establece una dinámica que contribuye a resolver los problemas y a comprender las prácticas que tienen lugar en la vida cotidiana de la escuela (p. 14).
Siguiendo las fases del modelo de Kemmis (1989), a continuación se transcribe y explícita los aspectos que se incluyeron en cada uno:

Ciclo 1. Este ciclo cuenta con las fases generales del proyecto, y es la base del proceso de investigación.

Planificación. Comprendió el planteamiento y la formulación del problema, así como el reconocimiento del contexto educativo, a través de la caracterización del cuerpo docente.

Acción. Se llevó a cabo con el diseño e implementación de estrategias en tres fases: exploración, estructuración y ejecución. En la primera, se realizaron actividades de sensibilización y motivación, y se establecieron las normas y mecanismos de participación en la investigación. En la segunda, se estudiaron los referentes teóricos sobre las situaciones problema, y se escogieron las situaciones a implementar con los estudiantes. En la tercera, se realizaron las sesiones de clase basadas en las situaciones problema.

Observación. Las docentes observaron sesiones de clase dirigidas por los investigadores sobre el desarrollo de las diferentes etapas propuestas para la solución de las situaciones problema.

Reflexión. Las docentes, luego de la terminación de cada etapa, diligenciaron un registro escrito reflexionando sobre los elementos didácticos relevantes para el aprendizaje de los estudiantes. Para la última etapa, se hizo una entrevista para todo el grupo colaborativo 
Consolidación y sistematización. Los investigadores analizaron las reflexiones hechas por las docentes, las sistematizaron y caracterizaron. de la investigación. Con base en las reflexiones, las docentes (re)significaron sus prácticas de aula.

\section{Consolidación y sistematización.}

Los investigadores analizaron las reflexiones hechas por las docentes, las sistematizaron y caracterizaron.

Ciclo 2. Con base en las (re) significaciones hechas, se replanteó el nuevo ciclo y se continuó con la espiral propuesta para ir mejorando los procesos de enseñanza y de aprendizaje.

\section{Población y muestra}

El conjunto total de individuos que conformaron la población de nuestra investigación, correspondió a 12 docentes que orientan el área de matemáticas en la sede primaria urbana. A través de un muestreo cualitativo intencionado, se escogieron cuatro docentes que orientan el área de matemáticas en la sede primaria urbana. Una docente con asignación de matemáticas por 20 horas semanales en el grado quinto, y tres docentes con asignación de matemáticas por 5 horas semanales, cada una en el grado tercero. Con la colaboración de las cuatro docentes junto a los dos investigadores del proyecto, se conformó el grupo colaborativo de investigación.

\section{Taller investigativo}

El taller investigativo se usó como técnica de recolección y análisis de información, se tuvieron en cuenta las cuatro fases planteadas por Quintana (2006): la primera permitió identificar y relacionar personalmente a los participantes, plantear los objetivos y metas del taller; la segunda se centró en la realidad objeto de análisis a través de la elaboración de unos registros escritos; en la tercera, se identificaron las líneas de acción; y en la última se estructuró y concertó el plan de trabajo (p. 147).

\section{Material de trabajo}

Se usó la propuesta del material del Programa Todos a Aprender 2.0 (PTA 2.0) del grupo Prest (MEN, 2015b), que promueve el desarrollo de la competencia matemática a partir de la resolución de problemas. Se utilizaron dos situaciones problema: en grado tercero "Se necesita un arquitecto para el zoológico" (MEN, 2016b), y para grado quinto "Un refugio para animales” (MEN, 2016c). Las dos situaciones presentan un problema en un contexto determinado que se le propone solucionar al estudiante. La situación problema se presenta con una secuencia didáctica ligada al enfoque de Resolución de Problemas (RdP) descrito por Pólya (MEN, 1998), que consta de cuatro fases: comprensión del problema, concepción de un plan, ejecución del plan y visión retrospectiva. Estas fases del enfoque RdP, se integran en el material seleccionado en cuatro etapas: comprensión, descontextualización (centros de aprendizaje), resolución y reflexión.

\section{Categorias de análisis en la resolución de las situaciones problema}

Teniendo en cuenta la relación del material de trabajo del grupo Prest 
(MEN, 2015b) con los elementos teóricos reconocidos en la investigación, en cuanto a: el concepto de situación problema (D’Amore, 2006), las fases para abordar un problema (MEN, 1998), el desarrollo de procesos del pensamiento matemático (MEN, 1998); se determinó acoger las cuatro etapas (comprensión, descontextualización, resolución y reflexión) propuestas

Tabla 1.

Categorías de análisis en la resolución de las situaciones problema

\begin{tabular}{lll}
\hline Categorías & Subcategorías & Instrumentos \\
\hline $\begin{array}{l}\text { Comprensión del } \\
\text { problema }\end{array}$ & $\begin{array}{l}\text { Estrategias } \\
\text { Contextualización } \\
\text { Plan de acción }\end{array}$ & $\begin{array}{l}\text { Registro escrito 1. Reflexión sobre la etapa } \\
\text { de comprensión del problema. Entrevista al } \\
\text { grupo colaborativo de la investigación. }\end{array}$ \\
$\begin{array}{l}\text { Descontextualización } \\
\text { (Centros de Aprendizaje). }\end{array}$ & $\begin{array}{l}\text { Exploración } \\
\text { Ejercitación } \\
\text { Aplicación }\end{array}$ & $\begin{array}{l}\text { Registro escrito 2. Reflexión sobre la } \\
\text { etapa de descontextualización (centros de } \\
\text { aprendizaje). Entrevista al grupo colaborativo } \\
\text { de la investigación. }\end{array}$ \\
$\begin{array}{l}\text { Resolución de la } \\
\text { situación problema. }\end{array}$ & $\begin{array}{l}\text { Aprendizajes } \\
\text { Trabajo colaborativo }\end{array}$ & $\begin{array}{l}\text { Registro 3. Reflexión sobre la etapa de } \\
\text { resolución de la situación problema. } \\
\text { Entrevista al grupo colaborativo de la } \\
\text { investigación. }\end{array}$ \\
& $\begin{array}{l}\text { Análisis de los tres registros escritos y de } \\
\text { la Entrevista al grupo colaborativo de la } \\
\text { Reflexión }\end{array}$ & $\begin{array}{l}\text { Actitudes } \\
\text { (re)Significación }\end{array}$ \\
& &
\end{tabular}

Comprensión de la situación problema. Se fundamenta en el uso de estrategias de comprensión y la preparación adecuada del contexto. Para facilitar la identificación de las tareas para resolver el problema, se construye un esquema de la situación problema, en donde se reconoce un plan de acción a seguir y los conceptos y procedimientos que necesitarán para solucionar la tarea y orientarlos en la organización de su trabajo (MEN, 2015b, p. 19). en la secuencia didáctica del material del grupo Prest, como categorías para analizar las reflexiones de las docentes frente a la aplicabilidad del uso de las situaciones problema y de la transposición didáctica realizada. Además, teniendo en cuenta los elementos más relevantes de cada etapa que se describen en los siguientes párrafos, se dedujeron las subcategorías objeto de análisis para la investigación.

Registro escrito 1. Reflexión sobre la etapa grupo colaborativo de la investigación.

Registro escrito 2. Reflexión sobre la etapa de descontextualización (centros de aprendizaje). Entrevista al grupo colaborativo de la investigación. resolución de la situación problema. Entrevista al grupo colaborativo de la investigación. la Entrevista al grupo colaborativo de la

Descontextualización (centros de aprendizaje). La situación problema presenta un reto para los estudiantes y genera en ellos la necesidad de aprender algo nuevo para poder resolverla. Los centros de aprendizaje son el escenario en donde se adquieren esos conocimientos, dejando de lado temporalmente el contexto de la situación problema (MEN, 2015b, p. 24). 
Resolución de la situación problema. Se inicia con un retorno al esquema de la situación problema realizado en la etapa de comprensión y un enriquecimiento del mismo a partir de los conceptos y procedimientos desarrollados durante los centros de aprendizaje. A continuación, cada estudiante diseña una estrategia de resolución para la cual debe definir un orden y una combinación apropiada de los conceptos y procedimientos adquiridos previamente. Finalmente, se comparten y contrastan las diversas estrategias de resolución y se procede a una validación de la solución (institucionalización). Esta etapa corresponde a la fase de ejecución del plan en las fases de RdP descritas por Pólya (MEN, 2015b, p. 38).

Reflexión. La última etapa consiste en un proceso de metacognición que se realiza colectivamente: los estudiantes, guiados por preguntas, reflexionan sobre lo aprendido y sobre su proceso de aprendizaje y toman conciencia de sus procesos mentales. Esta etapa facilita la transferencia de conocimientos en posibles situaciones futuras dentro y fuera del aula. La etapa de reflexión corresponde a la fase de visión retrospectiva descrita por Pólya (MEN, 2015b, p. 40).

Además, en la investigación la categoría de reflexión también hace referencia a los aspectos relevantes que las docentes reconocen en la implementación de la secuencia didáctica del material Prest del Programa Todos a Aprender, propuesta para solucionar situaciones problema.

\section{Estructura de los instrumentos de recolección de la información}

Se diseñaron tres registros escritos conformados por seis preguntas cada uno, y se realizó una entrevista al grupo colaborativo de la investigación con 10 preguntas orientadoras. El primer registro escrito se aplicó luego de implementar en el aula de clase con los estudiantes la etapa de comprensión de las situaciones problema; el segundo, al finalizar la etapa de descontextualización (desarrollo de los centros de aprendizaje); y el tercero, comprende la etapa de resolución de la situación problema.

La entrevista se aplicó al finalizar la ejecución de la secuencia didáctica, es transversal a las tres etapas anteriores, y también corresponde a la etapa de reflexión. Las docentes diligenciaron los registros escritos y contestaron la entrevista reflexionando sobre la efectividad de los elementos didácticos en la aplicación de las situaciones problema. Con base en las respuestas de las docentes, se elaboró una matriz de análisis en el programa Excel conformada por nueve columnas: categoría, pregunta, código docente, respuesta, codificación in vivo, codificación sustantiva, axial, selectiva y el análisis; y así determinar los aspectos más relevantes a los cuales las docentes hacían referencia.

Cada registro correspondió a una categoría de análisis con dos preguntas para sus respectivas subcategorías, así: Registro 1. Categoría: comprensión del problema, subcategorías: 
estrategias, contextualización y plan de acción; Registro 2. Categoría: descontextualización, subcategorías: exploración, ejercitación y aplicación; Registro 3. Categoría: resolución de la situación problema, subcategorías: aprendizajes, trabajo colaborativo y efectividad. La entrevista correspondió a las cuatro categorías: comprensión, descontextualización, resolución y reflexión, y a las subcategorías: preconcepciones, actitudes y (re) significación.

\section{Descripción del análisis y de la reflexión}

Análisis sobre la etapa de comprensión. Las docentes manifestaron que esta etapa propició que los estudiantes estuvieran motivados todo el tiempo, se integrará el trabajo realizado con otras áreas del conocimiento, que los estudiantes conocieran la estructura y forma de trabajo para desarrollar la situación problema. Con relación a esta etapa, la profesora (FA) dijo: "es como la base para desarrollarla, para poder desarrollar la situación del problema [...] y se sienten motivados todo el tiempo [...] porque ahí se trabajó matemáticas, se trabajó castellano, naturales, se trabajó vocabulario".

Frente a las estrategias de comprensión (contextualización, esquema, plan de acción) propuestas en la secuencia didáctica, las docentes consideraron que fueron una herramienta importante para comprender las tareas a desarrollar. La profesora (LU) exaltó: "son importantes porque primero en la etapa de comprensión el niño tiene que tener un como [...] o sea, entender lo que va a desarrollar".

Análisis sobre la etapa de descontextualización. Las docentes valoraron que el uso del material manipulativo en los centros de aprendizaje fue eficaz, posibilitando al estudiante alcanzar por sí mismo el aprendizaje, a través de una enseñanza activa, donde se aprende haciendo. Al respecto, la docente (FA) indica: "se logró, se aclaró y ellos entendieron exacto, en sí qué era área y como se hallaba, si, entonces fue muy beneficioso y muy efectivo para poder llegar a adquirir los aprendizajes" "Sí, porque ellos lograron llenar esos vacíos que tenían, porque sí había mucho error en los niños".

\section{Análisis sobre la etapa de resolución.}

Las docentes expresaron que los estudiantes adquirieron aprendizajes sobre área, perímetro, plano cartesiano, fracciones (grado tercero), y potenciación, radicación, fracciones, decimales, volumen, e interpretación de datos (grado quinto) a través de las diferentes actividades que se encuentran dentro de los centros de aprendizaje, desarrollando su pensamiento matemático. La docente (LU) expresa:

"Si me plantean primero una situación problema, y empiezo a buscar, o sea, me plantean un problema, y tengo que buscar estrategias, buscar herramientas, y buscar todo para llegar a solucionarlo, obviamente que está produciendo en mi estudiante, un pensamiento matemático; obviamente porque, estoy haciendo que él piense para que llegue a solucionarlo, obvio
Si me plantean primero una situación problema, y empiezo a buscar, o sea, me plantean un problema, y tengo que buscar estrategias, buscar herramientas, $\mathrm{y}$ buscar todo para llegar a solucionarlo, obviamente que está produciendo en mi estudiante, un pensamiento matemático 
que, yo lo entiendo así, tiene que haber un pensamiento matemático [...] por ejemplo Daniel, cuando entraba y lo planteaba de varias formas, de dos o tres formas diferentes a todos sus compañeros, había pensamiento matemático en la forma como él lo solucionaba".

Además, las docentes manifestaron que el trabajo en grupo es una buena estrategia para el desarrollo de las diferentes actividades, porque los estudiantes lograron organizarse, colaborarse entre pares y grupos, también fueron capaces de trabajar mancomunadamente en busca de dar solución a la situación problema. Al respecto, la docente (LE) dijo:

"Un aspecto es el trabajo en grupo, en la clase de matemáticas nunca se trabaja en grupo [...] en ésta situación problema los estudiantes organizan sus grupos, el uno se apoya del otro, se organizan los grupos de trabajo, y de verdad, excelente [...] los niños sobresalen unos con otros [...] ahí en grupo uno ya, ve avances en los niños, y cosas que no sabíamos de muchos niños, de participar, de hablar, desenvolverse, de solucionar un problema [...] los niños de verdad, los que nunca habían hablado, ahí hablaron, participaron”.

La aceptación de la aplicación de la secuencia didáctica para solucionar las situaciones problema, fue unánime. La docente (FA) expone: "Claro, que se siga una secuencia, claro, sería muy bueno, coger los centros de aprendizaje como actividades no generan resultado, ni aprendizaje, claro que no. Es importante realizar todos los pasos que vienen ahí"; la profesora (LE) manifestó: "Los centros de aprendizaje no se pueden trabajar desligados, deben ser el punto principal, para poder, no puede retirarse del, o sea desligarse de la situación problema"; la docente (LU) definió: "Es un proceso que da un resultado, totalmente, un resultado. Lo más conveniente es trabajar toda la secuencia didáctica, sí claro”.

\section{Análisis sobre la etapa de reflexión.} Con relación a las preconcepciones de las docentes, ellas manifestaron que antes también se tenían en cuenta los presaberes, pero los problemas se planteaban para todo el curso, se estructuraban y planteaban en el tablero, se desarrollaban de manera mecánica. La docente (LU) frente a la diferencia entre la forma como desarrollaban las situaciones problema antes de la investigación, afirma:

"No, era totalmente diferente, pero si se planteaba el problema, o sea un problema para todo el curso que se podía estructurar y plantear en el tablero, era para todos los estudiantes, pero yo reconozco que este trabajo tiene cosas que hacen pensar al niño, que lo obliga a que lea, entienda lo que le están preguntando y pueda desarrollar la parte matemática, pero interpretando, cosa que antes tal vez antes se hacía menos práctica, y más directamente, como desarrollarla más mecánica. ¿Cómo divide? es la división, entonces uno se limitaba a tengo que hacer la división, desarrolle el proceso de la operación y doy una respuesta. Aquí, ponen al niño de pronto más incógnitas a que él intérprete, desarrolle y aplique lo que aprende". 
Las docentes identificaron que los estudiantes estuvieron motivados, interesados, $\mathrm{y}$ trabajando en equipo con el ánimo de desarrollar las actividades propuestas en la situación problema. La docente (FA) manifestó:

"Un aspecto es la motivación que tuvieron, el material, las ganas de querer todos participar, sí, de todos manipular su material, de todos poder solucionar el problema, e, que (sic) más relevante, el comportamiento pues, el comportamiento fue bueno porque estaban todos motivados, trabajando todos para lograr solucionar su problema".

A través de las reflexiones, las docentes consideraron (re)significar sus prácticas de aula en la iniciación con una situación particular, las estrategias y subetapas de comprensión, la contextualización, el uso de material manipulable. La docente (FA), expresa:

"De verdad que sí podemos retomar muchas cosas, por ejemplo la iniciación con el cuento, la comprensión ¿sí? Porque por ejemplo en lo del zoológico fue muy motivante la comprensión lectora del cuento, los pasos que hay que seguir para tratar de hacer una buena contextualización, a bregar a retomar lo que uno más pueda ¿̇sí? y el material pues si se pudiera llevar, pues sería una maravilla, porque de verdad uno con material donde los niños manipulen es lo mejor, donde manipulen jueguen y de verdad llevan un buen aprendizaje y hasta los estudiantes que son como los más, los que andan como más distraídos, algo los motiva, algo pero bueno aquí tal cosa, aquí tal otra, ¿sí? algo les quedó en sus cabezas, entonces si es muy bueno. El hecho de que participen porque hay unos niños que ni hablaban fue motivante, porque hasta los que no participan hablaban".

También las docentes reconocen que la aplicación de la situación problema permitió la participación de todos los estudiantes, haciendo que disminuyera la mortalidad académica. La docente (LE) expone:

"Yo pienso que con esta metodología, así con esa didáctica ningún niño me pierde matemáticas, ninguno y como lo hacemos nosotros normalmente hay mortalidad académica en matemáticas, claro que me ha servido mucho, profe... lo trabajó en quinto $\mathrm{A}$, yo lo multiplique en 5B, 5C y 5D; de ahí que en el tercer periodo casi nadie perdió matemáticas, como 4 o 5 estudiantes nada más perdió matemáticas, entonces los niños que uno tenía como candidatos a perder ya no, porque sí, ya por lo menos uno dice hombre, uno muchas veces comete muchos errores, como docente comete errores y hay niños que uno no les da la oportunidad, y con esta secuencia didáctica uno encuentra en los estudiantes algo bonito, y todos los niños saben y quieren aprender".

Las docentes reconocen que se debe identificar y saber que todos aprenden de acuerdo con sus intereses, que la base de todo es el juego, la relevancia del trabajo en grupo, que todo el curso es un grupo. La docente (LU) dice:

"Identificar y saber que todos aprenden de acuerdo a sus intere- 
ses, ya que se sienten motivados [...] la base de todo es el juego, jugando se aprende todo, el trabajo grupal no haciendo un grupo, sino un grupo en toda la aula de clase, un grupo completo, en el que cada uno aportaba, el grupo era el salón entero y todos los niños querían hablar, querían coger el material y tenían la oportunidad todos, y demostrar que todos podían y todos pueden”.

\section{Resultados}

Con el análisis de los resultados de la investigación, se cumplió con el objetivo general propuesto, debido a que se pudo caracterizar los aspectos relevantes en la reflexión que hicieron las docentes sobre las prácticas de aula al implementar situaciones problema para desarrollar el pensamiento matemático en los estudiantes de tercero y quinto de la sede primaria urbana. Todo lo anterior se soporta a continuación con los resultados generales de la investigación.

En la fundamentación teórica de la investigación, se establecieron algunos aspectos didácticos relevantes sobre el uso de las situaciones problema, que sirvieron como base para el fortalecimiento didáctico del contenido de las docentes, y para la selección del material a trabajar con los estudiantes.

El material que se seleccionó para implementar en el aula las situaciones problema, correspondió al elaborado por el grupo Prest del Programa Todos a Aprender 2.0. (MEN, 2016b, 2016c), teniendo en cuenta su relación con el concepto sobre situación problema, las etapas para abordar un problema y los procesos de desarrollo del pensamiento matemático, establecidos en los aspectos didácticos de la investigación.

Con el análisis sobre las reflexiones de las docentes en la etapa de comprensión (registro 1 y entrevista), se evidencia que ellas reconocen que las estrategias de comprensión aplicadas fueron pertinentes y beneficiosas para los estudiantes, ya que permitieron que ellos reconocieran las tareas a resolver, los pasos a seguir, y los conceptos y procedimientos que se necesitaban para solucionar la situación problema.

En la etapa de descontextualización (centros de aprendizaje), las docentes a través de sus escritos en los registros, y de sus afirmaciones expresadas en la entrevista, evidencian que en el desarrollo de los centros, los estudiantes construyeron y afianzaron sus conceptos, desarrollando procesos matemáticos con el uso de material manipulativo; siendo relevante el trabajo en equipo, la socialización de los trabajos y la participación de todos los estudiantes para la consecución de los aprendizajes. También, identificaron que la orientación de los centros de aprendizaje se basó en una enseñanza activa, donde se aprendió haciendo, destacando el uso de actividades lúdicas (juego), el acceso de todos los niños al material y la realización de las actividades por parte de todos los estudiantes, aclarando sus dudas y comprendiendo los temas trabajados.

Las docentes en sus reflexiones manifestaron que a través del desarrollo de las actividades propuestas en las etapas 
de comprensión, descontextualización y resolución de la situación problema, los estudiantes adquirieron aprendizajes sobre área, perímetro, plano cartesiano $\mathrm{y}$ fracciones en grado tercero; $\mathrm{y}$ potenciación, radicación, fracciones, decimales, volumen, e interpretación de datos en grado quinto, desarrollando su pensamiento matemático $\mathrm{y}$ demostrando la comprensión de las situaciones problema al darles solución.

Un aspecto que prevalece en las reflexiones de las docentes como importante para el buen desarrollo de las actividades propuestas, que evidenciaron en las diferentes etapas de la resolución de la situación problema, es el trabajo en grupo de manera colaborativa, ya que ellas reconocen que los estudiantes aportaron sus saberes, aclararon dudas mutuamente, se organizaron y fueron capaces de trabajar mancomunadamente en busca de dar solución a la situación problema.

Las docentes a través de las respuestas en la entrevista, se mostraron de acuerdo con la efectividad que trae consigo desarrollar toda la secuencia didáctica (etapas de comprensión, descontextualización, resolución y reflexión) para solucionar las situaciones problema, al reconocer que el trabajo realizado durante las diferentes etapas coadyuvó a los estudiantes en la resolución de las situaciones problema, facilitando la comprensión del mismo y la adquisición de aprendizajes, mantuvieron la participación y el interés de los estudiantes, convirtiéndose en un ambiente propicio para construir conceptos y aplicarlos en ejercicios prácticos. También, recomiendan y aseguran que se deben implementar todas las etapas de la secuencia didáctica para que se puedan obtener mejores resultados y alcanzar los objetivos de aprendizaje.

En las respuestas de las docentes a la entrevista, se vislumbra que tenían poco conocimiento didáctico sobre el uso de las situaciones problema en el aula, debido a que se percibe que usualmente desarrollaban problemas rutinarios con los estudiantes, lo que los impulsaba a procesos de enseñanza y aprendizaje mecánicos. Las docentes no profundizaron en las respuestas sobre las concepciones que tenían acerca de las situaciones problema, pero sí expusieron varios beneficios con la secuencia didáctica ejecutada en la investigación y evidenciados en los análisis de los resultados.

Un aspecto fundamental para la consecución de los logros planteados a través de la resolución de las situaciones problema, destacada en las reflexiones de las docentes, fue la actitud de los estudiantes, la cual se reflejó en las diferentes etapas ejecutadas de la secuencia didáctica, como sobresaliente, debido en gran parte a la motivación permanente, el interés, el trabajo individual, el trabajo en grupo, el trabajo colaborativo, la responsabilidad, y la participación.

Durante el desarrollo de la investigación y con base en las reflexiones de las docentes evidenciadas en los análisis de los relatos escritos y orales por medio de los instrumentos de recolección de la información, se reconoce que las
Un aspecto que prevalece en las reflexiones de las docentes como importante para el buen desarrollo de las actividades propuestas, que evidenciaron en las diferentes etapas de la resolución de la situación problema, es el trabajo en grupo de manera colaborativa

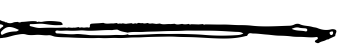


docentes iniciaron un proceso de (re) significación de sus prácticas de aula, relacionado con la implementación de las situaciones problema, principalmente resaltan: el identificar los intereses de los estudiantes, la iniciación con una situación particular, las estrategias y subetapas de comprensión, la contextualización, el uso de material manipulable, el juego como base, el trabajo en grupo, la preparación de clase integrando varios temas y la participación de todos los estudiantes. Además, manifestaron que la forma como se trabajó con las situaciones problema, hizo que disminuyera la reprobación académica del área por parte de los estudiantes.

Con los acompañamientos de aula que los investigadores hicieron en el desarrollo de las situaciones problema, se observó que los niños desarrollaron las actividades de todas las etapas de la secuencia didáctica, pero que tuvieron un mejor desempeño en los centros de aprendizaje, especialmente con el uso del material manipulativo; y mayor dificultad en la etapas de comprensión y resolución de la situación problema, especialmente en las actividades de proposición individual. Adicionalmente, en la etapa de reflexión, la mayoría de los estudiantes no lograron hacer el proceso de metacognición que propone la secuencia didáctica.

\section{Conclusiones}

La investigación, en el ámbito de la formación de profesores, orientados por la conformación de comunidades académicas en contextos colaborativos y la reflexión sobre las actividades de aula con sus estudiantes, para su posterior (re)significación; permitió detectar los siguientes aspectos relevantes, como contribución de esta búsqueda permanente de sentido, intersubjetiva, de la actividad docente en el aula.

El uso de las situaciones problema para desarrollar el pensamiento matemático, tiene un fundamento teórico $\mathrm{y}$ pragmático que fortalece las prácticas de aula por parte del docente, y facilitan la comprensión de los procesos matemáticos en los estudiantes. Para el caso de la investigación, las situaciones problema "un refugio de animales" y "se necesita un arquitecto para el zoológico", constituyeron un ambiente para propiciar el acercamiento de los estudiantes al conocimiento de su contexto, y a las docentes como oportunidad para analizar los aspectos didácticos que influyen el aprendizaje de los estudiantes.

Las situaciones problema desarrolladas facilitaron la integración en los planes de aula/clase y en el desarrollo de la sesiones de clase, de los pensamientos espacial, aleatorio y métrico en torno al pensamiento numérico, y el aprendizaje de las operaciones con fracciones y conceptos como el perímetro, área y volumen.

El proceso llevado a cabo en la resolución de las situaciones problema, por medio de la secuencia didáctica planteada en las cartillas del grupo PREST del programa Todos a Aprender del MEN, lleva a los estudiantes a la 
aproximación y uso de los conceptos matemáticos mencionados, a través de la vivencia partiendo de lo manipulativo (concreto y realista), pasando a lo pictórico y finalmente lo simbólico.

Aunque la implementación de las situaciones problema fueron pertinentes y beneficiosas, es necesario reconocer que la efectividad de esta propuesta en el aprendizaje de las matemáticas por parte de los estudiantes, radicó en gran parte en la transposición didáctica de la secuencia planteada por el material del grupo PREST del Programa Todos a Aprender del MEN, llevada a cabo por los investigadores y las docentes del grupo colaborativo.

Como aspectos relevantes que hicieron exitoso el proceso llevado a cabo en el aprendizaje de los estudiantes, se identifican: el uso de material manipulativo, la vinculación individual y consciente de los estudiantes desde la etapa de comprensión de la situación problema, la motivación e interés permanente en el desarrollo de las diferentes sesiones de clase, el trabajo colaborativo, la realimentación y la participación constante.

Las etapas propuestas por el material seleccionado, a través de la secuencia didáctica, soporta un fundamento pedagógico y didáctico coherente, que permite con el buen desarrollo de las actividades propuestas, la consecución de las metas de aprendizaje que se plantean en las situaciones problema, y por ende, el desarrollo de competencias matemáticas en los estudiantes.
Los procesos de enseñanza y de aprendizaje que se desarrollan en el aula, están sujetos, en gran medida, a la experticia de los docentes por generar ambientes propicios para el desarrollo de competencias. Esta capacidad profesional se fortalece si el docente profundiza su conocimiento didáctico del contenido y es capaz de trabajar colaborativamente con sus pares académicos.

Con la investigación acción realizada, se logró fortalecer procesos institucionales, como la actualización de los planes de área, elaboración de planes de aula, reuniones de discusión y toma de decisiones, y la reflexión intersubjetiva sobre las prácticas implementadas.

La reflexión de las docentes sobre las prácticas de aula, les permitió (re) significar algunos aspectos didácticos sobre el uso de las situaciones problema para desarrollar el pensamiento matemático en los estudiantes, como el dar mayor atención a los intereses de los estudiantes con situaciones particulares que les llamen la atención, el integrar varios pensamientos en torno a una misma situación, la relevancia de las orientaciones en el trabajo individual y en grupo, el manejo de estrategias de comprensión, la contextualización de las situaciones problema, la elaboración de esquemas, y el seguimiento de etapas para la resolución de las situaciones problema.

Las docentes reconocieron las diferencias en el trabajo con situaciones problema de los problemas usualmente trabajados en el aula, debido entre otras
Con la investigación acción realizada, se logró fortalecer procesos institucionales, como la actualización de los planes de área, elaboración de planes de aula, reuniones de discusión y toma de decisiones, y la reflexión intersubjetiva sobre las prácticas implementadas. 
cosas, al contexto en el que se desarrolla el problema, la relevancia en el aporte de los estudiantes para la resolución del mismo, y las diferentes estrategias didácticas que se trabajaron durante las etapas de la secuencia didáctica.

La reflexión es un proceso interior, que cada individuo logra dependiendo principalmente de su voluntad, sin embargo, hay factores externos que propician esta práctica reflexiva, como son los espacios con ambientes propicios para expresarse libremente, donde el individuo se sienta escuchado y valorado, y se construya socialmente y se empodere tanto al maestro como el grupo docente.

El análisis que los investigadores hicieron sobre los procesos de reflexión al interior del grupo, permitió caracterizar elementos importantes para la formación continua del grupo de docentes, como el uso de situaciones problema, la efectividad de la secuencia didáctica, el trabajo con material manipulativo, la evaluación formativa, la metodología COPISI (concreto, pictórico y simbólico), la transposición didáctica, los procesos de metacognición, entre otros.

Para los investigadores, constituyó una experiencia fascinante respecto a las estrategias de conformación de comunidades académicas y de práctica en ámbitos colaborativos, que permitió el intercambio de experiencias entre docentes, que propició crecer profesionalmente y desarrollar competencias respecto a la reflexión intersubjetiva de situaciones de aula, con miras a mejorar el aprendizaje de la matemática a nivel institucional, aspecto considerado como el mayor aporte de la investigación, el crecimiento profesional y desarrollo de procesos de reflexión de docentes, investigadores y estudiantes, lo cual permitió una evidente mejora en la calidad educativa de la Institución involucrada.

\section{Referencias bibliográficas}

D'Amore, B. (1997). Problemas. Pedagogía y psicología de la matemática en la actividad de resolución de problemas. Madrid, España: Editorial Síntesis, S.A.

D’Amore, B. (2006). Didáctica de la matemática. Bogotá, Colombia: Didácticas Magisterio.

D'Amore, B., Fandiño, M., Marazzani, I., \& Sbaragli, S. (2007). La didáctica y la dificultad matemática. Análisis de situaciones con falta de aprendizaje. Bogotá, Colombia: Didácticas Magisterio.

Jiménez, A. (2005). Formación de profesores de matemática: aprendizajes recíprocos. Escuela - Universidad. Tunja: Universidad Pedagógica y Tecnológica de Colombia. 
Ministerio de Educación Nacional (MEN) (1998, 7 de junio). Serie lineamientos curriculares. Matemáticas. Santa Fe de Bogotá, D.C., Colombia.

Ministerio de Educación Nacional (MEN) (2005). Estándares básicos de competencias en matemáticas. Potenciar el pensamiento matemático: jun reto escolar!

Ministerio de Educación Nacional (MEN) (2015a). Siempre día E. Informe por colegio Pruebas Saber $3^{\circ} 5^{\circ}$ y $9^{\circ}$. Aterrizando los resultados al aula. Santana, Boyacá: Colegio Antonio Ricaurte.

Ministerio de Educación Nacional (MEN) (2015b). Documento Maestro. Guía de enseñanza para docentes de primaria. Grado tercero. PREST.

Ministerio de Educación Nacional (MEN) (2016a). Siempre día E. Informe por colegio Pruebas Saber $3^{\circ} 5^{\circ}$ y 9․ Aterrizando los resultados al aula. Santana, Boyacá: Colegio Antonio Ricaurte.

Ministerio de Educación Nacional (MEN) (2016b). Programa para la excelencia docente y académica, Todos a Aprender 2.0. Situación 4-5. Grado tercero. PREST.

Ministerio de Educación Nacional (MEN) (2016c). Programa para la excelencia docente y académica, Todos a Aprender 2.0. Situación 4-5. Grado quinto. PREST.

Murillo, F. (2011). Investigación - Acción. Métodos de investigación en educación especial. 3ㅁ. Educación Especial.

Perrenoud, P. (2007). Desarrollar la práctica reflexiva en el oficio de enseñar. Profesionalización y razón pedagógica. México, D.F., México: Colofón, S.A.

Plan de área de matemáticas (IEAR, 2017). Horizonte Institucional. Institución Educativa Técnica Antonio Ricaurte. Santana, Boyacá.

Quintana, A. (2006). Metodología de Investigación Científica Cualitativa. En A. Quintana \& W. Montgomery (Eds.), Psicología: Tópicos de actualidad (pp. 4784). Lima, Perú.

Sandoval, C. (1996). Investigación cualitativa. En Instituto Colombiano para el fomento de la Educación Superior (ICFES) (Ed.), Programa de Especialización en Teoría, Métodos y Técnicas de Investigación Social (pp. 1-313). Bogotá, Colombia: Arfo Editores e Impresores Ltda. 